\title{
Role of compensatory meiosis mechanisms in human spermatogenesis
}

\author{
Mareike Borgers, Martin Wolter, Anna Hentrich, Martin Bergmann, Angelika Stammler and \\ Lutz Konrad \\ Department of Obstetrics and Gynecology, Medical Faculty, Feulgenstraße 12, D-35392 Giessen, Germany and \\ ${ }^{1}$ Institute of Veterinary-Anatomy, -Histology and -Embryology, Frankfurter Straße 98, D-35392 Giessen, Germany \\ Correspondence should be addressed to L Konrad; Email: Lutz.Konrad@gyn.med.uni-giessen.de
}

\begin{abstract}
Disturbances of checkpoints in distinct stages of spermatogenesis (mitosis, meiosis, and spermiogenesis) contribute to impaired spermatogenesis; however, the efficiency of meiotic entry has not been investigated in more detail. In this study, we analyzed azoospermic patients with defined spermatogenic defects by the use of octamer-binding protein 2 for type A spermatogonia, sarcoma antigen 1 for mitosis-meiosis transition and SMAD3 for pachytene spermatocytes. Especially patients with maturation arrest (MA) at the level of primary spermatocytes showed significantly reduced numbers of spermatogonia compared with patients with histologically intact spermatogenesis or patients with hypospermatogenesis (Нyp). For a detailed individual classification of the patients, we distinguished between 'high efficiency of meiotic entry' (high numbers of pachytene spermatocytes) and 'low efficiency of meiotic entry' (low numbers of pachytene spermatocytes). Only patients with histologically normal spermatogenesis (Nsp) and patients with Hyp showed normal numbers of spermatogonia and a high efficiency of meiotic entry. Of note, only patients with histologically Nsp or patients with Hyp could compensate low numbers of spermatogonia with a high efficiency of meiotic entry. In contrast, patients with MA always showed a low efficiency of meiotic entry. This is the first report on patients with impaired spermatogenesis, showing that half of the patients with Hyp but all patients with MA cannot compensate reduced numbers in spermatogonia with a highly efficient meiosis. Thus, we suggest that compensatory meiosis mechanisms in human spermatogenesis exist.
\end{abstract}

Reproduction (2014) 148 315-320

\section{Introduction}

In about half of infertile couples, male infertility plays a role and almost $30 \%$ are caused by a male factor alone (Brugh \& Lipshultz 2004). Besides analysis of semen parameters, a histological evaluation is indicated especially to confirm obstructive azoospermia in men with testes of normal size and normal levels of reproductive hormones (Dohle et al. 2012).

The dynamics of spermatogenesis are crucial for elucidation of impaired spermatogenesis, which is caused by many factors such as X-ray, chemotherapy, infections, chromosomal defects, diabetes mellitus, or alcohol abuses (Cerilli et al. 2010). Although a lot of aberrations during mitosis (Steger et al. 1998, Bar-Shira Maymon et al. 2003) and meiosis (Gonsalves et al. 2004, Egozcue et al. 2005) have been described, only recently it has been shown that also reduced numbers of Sertoli cells or spermatogonia (including the stem cells) contribute to impaired spermatogenesis (Hentrich et al. 2011). However, in humans the stem cell niche is still poorly characterized, but $A_{\text {dark }}$ as well as $A_{\text {pale }}$ spermatogonia seem to contain subpopulations of spermatogonial stem cells (Dym et al. 2009). Of note, in primates, the $A_{\text {pale }}$ spermatogonia also contain few spermatogonial stem cells (Hermann et al. 2010).

The testicular stem cells in rat and mouse are localized nearly exclusively in a stem cell niche adjacent to the interstitium (Chiarini-Garcia et al. 2001, 2003). The stem cell niche is characterized by a microenvironment which is influenced also by Leydig cells and blood vessels (Shetty \& Meistrich 2007, Yoshida et al. 2007). Furthermore, also Sertoli cells and peritubular cells contribute to the self-renewal of the spermatogonial stem cells (Dadoune 2007, de Rooij 2009).

Especially in patients with maturation arrest (MA) a significant reduction in dividing spermatogonia during mitosis was observed (Steger et al. 1998, Bar-Shira Maymon et al. 2003), whereas in hypospermatogenesis (Hyp)-accelerated apoptosis rather than proliferative dysfunction was suggested to be responsible for reduced spermatogonial cell numbers (Takagi et al. 2001). In addition, in cases with MA reduced recombination rates (up to $50 \%$ ) were found (Gonsalves et al. 2004, Egozcue 
Table 1 Antibodies used for quantification of distinct germ cell types.

\begin{tabular}{|c|c|c|c|c|c|}
\hline Protein & Source & Catalog number & Species & Clonality & Dilution \\
\hline OCT2 & Novocastra (Wetzlar, Germany) & NCL-OCT-207 & Mouse & Monoclonal & $1: 75$ \\
\hline SAGE1 & Sigma-Aldrich (St. Louis, USA) & HPA003033 & Rabbit & Polyclonal & $1: 2000$ \\
\hline SMAD3 & EnoGene Biotech (Frankfurt, Germany) & E021324 & Rabbit & Polyclonal & $1: 300$ \\
\hline
\end{tabular}

et al. 2005) and an increased frequency of genetic abnormalities (Weedin et al. 2011).

In this study, we analyzed germ cell numbers in the early phase of spermatogenesis with an emphasis on mitosis-meiosis transition. Furthermore, we classified the deficiencies individually by distinguishing between high and low efficiency of meiosis. Our main finding in this study was that cases with histologically normal spermatogenesis (Nsp) and cases with Hyp could compensate reduced numbers of spermatogonia with a highly efficient meiosis suggesting a compensatory mechanism.

\section{Materials and methods}

\section{Patients}

Testicular biopsies were obtained from 90 patients between 1998 and 2012 and were indicated because of normogonadotropic obstructive or non-obstructive azoospermia. After written informed consent, biopsies were taken under general anesthesia. The Ethics Committee of the Medical Faculty of the Justus-LiebigUniversity, Giessen, Germany approved the study (75/00 and 56/05). After fixation in Bouins solution and embedding in paraffin, $5 \mu \mathrm{m}$ sections were stained with hematoxylin and eosin and spermatogenesis was evaluated histologically according to the scoring system of Bergmann \& Kliesch $(1998,2010)$. The patients were classified into histologically $\mathrm{Nsp}(n=30$; median age 39, range 16-56; mean score 9.93, range 9-10), Нyp ( $n=30$; median age 37 , range 18-63; mean score 8.43 , range $6-10$ ), and MA at the level of spermatocytes (MA, $n=30$; median age 34.5, range 17-69; mean score 0).

\section{Antibodies}

To classify especially early stages of spermatogenesis (spermatogonia and mitosis-meiosis transition), we identified several highly robust markers with a good signal-to-noise ratio and distinct expression pattern in human germ cells. For a detailed analysis of impaired spermatogenesis, three markers were chosen (Table 1), octamer-binding protein 2 (OCT2 (POU2F2)) for early spermatogonia (Lim et al. 2011), sarcoma antigen 1 (SAGE1) for dividing and differentiating spermatogonia (Lim et al. 2011), and mitosis-meiosis transition (Looijenga 2011), and SMAD3 for pachytene spermatocytes (Hentrich et al. 2011).

\section{Immunohistochemistry}

Immunohistochemistry was carried out on $5 \mu \mathrm{m}$ sections of bouin-fixed, paraffin-embedded specimen, as published previously (Konrad et al. 2007). The Envision System from Dako (Hamburg, Germany) combined with DAB staining was used according to the manufacturer's instructions. Counterstaining was done with hematoxylin. The antibodies used for detection are given in Table 1. Digital images were obtained with the inverse microscope FSX100 (Olympus) using the Olympus FSX-BSW Software. The images were processed with Adobe Photoshop 7.0.

\section{Quantification of germ cells}

The numbers of germ cells were obtained from $26.63 \pm 12.58$ tubular cross sections per specimen representing Nsp, Hyp, and MA for each staining. As published recently (Hentrich et al. 2011) and also shown in this study, it was sufficient to use 15-35 nearly round cross sections for quantification; we never observed outliers.

There is no consensus on how quantification of testicular biopsies with an emphasis on impaired spermatogenesis should be performed. The number of Sertoli cells is different in patients

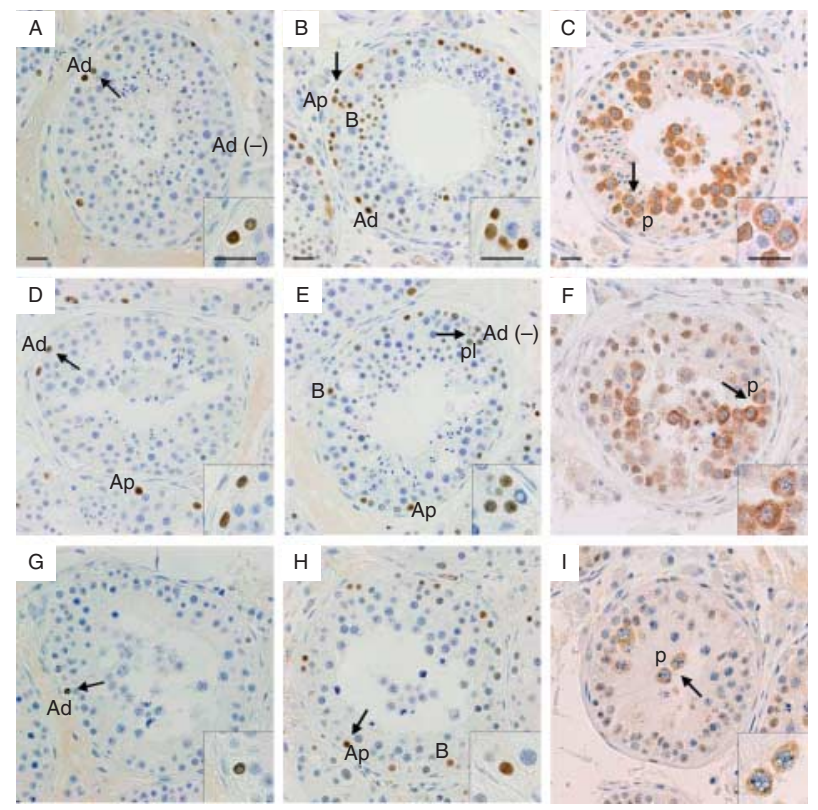

Figure 1 Immunohistochemical detection of OCT2 (A, D and G), SAGE1 ( $B, E$ and $H)$, and SMAD3 (C, F and $\mathrm{I})$ in biopsies from patients with histologically normal spermatogenesis (A, B and $C)$, patients with hypospermatogenesis $(\mathrm{D}, \mathrm{E}$ and $\mathrm{F})$, and cases with maturation arrest $(\mathrm{G}, \mathrm{H}$ and $\mathrm{I})$. OCT2 was found mainly in $\mathrm{A}_{\text {dark }}$ spermatogonia (Ad) and to a lesser extent in $A_{\text {pale }}$ spermatogonia (Ap). Also few OCT2-negative $A_{\text {dark }}$ spermatogonia $(\operatorname{Ad}(-))$ could be identified. SAGE1 was expressed in $A_{\text {dark }}$ spermatogonia up to type $B$ spermatogonia (B), but also in some (pre)-leptotene spermatocytes (pl). In contrast, SMAD3 was localized primarily in the cytoplasm of pachytene spermatocytes ( $p$ ). The arrows point to the regions provided enlarged in the insets. Scale bar $=25 \mu \mathrm{m}$ (A, B and C), the same magnification was used in (D, E, F, G, H and I). Counterstaining was done with hematoxylin. 
Table 2 Numbers of distinct germ cells per cross-section in impaired spermatogenesis

\begin{tabular}{|c|c|c|c|c|c|}
\hline & \multirow[b]{2}{*}{$\boldsymbol{n}$} & \multirow[b]{2}{*}{ Mean (S.E.M.) } & \multicolumn{3}{|c|}{$P$ values } \\
\hline & & & $\begin{array}{l}\text { Nsp vs } \\
\text { Hyp }\end{array}$ & $\begin{array}{c}\text { Nsp vs } \\
\text { MA }\end{array}$ & $\begin{array}{c}\text { Hyp vs } \\
\text { MA }\end{array}$ \\
\hline $\begin{array}{l}\text { OCT2: Early } \\
\text { spermatogonia }\end{array}$ & & & $\mathrm{ns}$ & 0.01 & 0.001 \\
\hline Nsp & 30 & $2.09(0.25)$ & & & \\
\hline Hyp & 30 & $2.05(0.23)$ & & & \\
\hline$M A$ & 30 & $0.96(0.16)$ & & & \\
\hline $\begin{array}{l}\text { SAGE1: dividing/ } \\
\text { differentiating } \\
\text { spermatogonia }\end{array}$ & & & 0.01 & 0.001 & ns \\
\hline Nsp & 30 & $15.62(0.82)$ & & & \\
\hline Hyp & 30 & $11.16(0.61)$ & & & \\
\hline $\mathrm{MA}$ & 30 & $9.10(0.77)$ & & & \\
\hline $\begin{array}{l}\text { SMAD3: pachytene } \\
\text { spermatocytes }\end{array}$ & & & 0.001 & 0.001 & 0.001 \\
\hline Nsp & 30 & $16.35(0.92)$ & & & \\
\hline Нур & 30 & $8.64(0.56)$ & & & \\
\hline$M A$ & 30 & $1.53(0.36)$ & & & \\
\hline
\end{tabular}

Nsp, normal spermatogenesis; Hyp, hypospermatogenesis; MA, maturation arrest; ns, not significant.

with MA or Hyp (Hentrich et al. 2011). Thus, the Sertoli cell to germ cell ratio is not applicable for the quantification of germ cell numbers.

\section{Statistical analysis}

Values from each experiment were used for calculation of the means (per cross section) and the respective S.E.M. Differences between the groups were calculated with the test from MannWhitney. The $P$ values below 0.05 were considered statistically significant.

Cut off values to distinguish between normal germ cell numbers vs reduced germ cell numbers were determined with receiver operating characteristic-curves and area under the curve. Besides sensitivity and specificity also the positive likelihood ratio was calculated. For all statistical analyses GraphPad Prism 6 (San Diego, CA, USA) was used.

\section{Results}

\section{Identification of germ cell markers for early spermatogenesis}

We identified the expression of OCT2 mainly in spermatogonia $A_{\text {dark, }}$ but also in very few spermatogonia
$A_{\text {pale }}$ (Fig. 1A, D and G). Often the OCT2-positive spermatogonia were localized in small groups in close proximity to interstitial arterioles or venules, which is in accordance to the characteristics of the stem cell niche in murine testis (Shetty \& Meistrich 2007, Yoshida et al. 2007) and thus have been also termed as reserve stem cells (Dym et al. 2009). However, we found also OCT2-negative $A_{\text {dark }}$ and $A_{\text {pale }}$ spermatogonia.

We identified SAGE1 in very few spermatogonia $A_{\text {dark, }}$ but in nearly all $A_{\text {pale }}$ and type $B$ spermatogonia as well as in (pre)-leptotene spermatocytes and round spermatids (Fig. 1B, E and $\mathrm{H}$ ), thus representing mitotic spermatogonia and transition of mitosis into meiosis. For characterization of pachytene spermatocytes, we used SMAD3 (Fig. 1C, F and I).

\section{Analysis of meiosis efficiency}

All three markers were used for the quantification of germ cell numbers (early spermatogenesis) per cross section and thus also for individual classification of meiosis efficiency in cases of impaired spermatogenesis. Quantification of OCT2-positive spermatogonia showed nearly identical values for Nsp and Hyp, but cases with MA demonstrated a significant reduction of $\sim 50 \%$ (Table 2). Of note, quantification of SAGE1-positive germ cells (spermatogonia up to (pre)-leptotene spermatocytes) revealed strongly and significantly reduced cell numbers in Hyp and in patients with MA compared with the controls with histologically Nsp (Table 2). Similarly, SMAD3-positive pachytene spermatocytes were reduced by $47 \%$ in Hyp and by $91 \%$ in cases with MA compared with Nsp (Table 2 ).

\section{Individual classification of patients with impaired spermatogenesis}

For a detailed individual classification of the patients, we determined the cut off values for all three markers to distinguish between normal and lower germ cell numbers (Table 3). Furthermore, we classified spermatogenesis into 'high efficiency of meiotic entry' (high numbers of SMAD3-positive spermatocytes, Fig. 2A) and 'low efficiency of meiotic entry' (low numbers of SMAD3-positive spermatocytes, Fig. 2B). Both groups could be further divided into four subgroups according

Table 3 Statistical analysis of the three markers used for individual classification.

\begin{tabular}{|c|c|c|c|c|c|c|c|c|c|}
\hline & \multicolumn{3}{|c|}{ OCT2 $($ Cut off $=1.06)$} & \multicolumn{3}{|c|}{ SAGE1 $($ Cut off $=10.84)$} & \multicolumn{3}{|c|}{ SMAD3 $($ Cut off $=8.6)$} \\
\hline & Sensitivity & Specificity & $\mathrm{LR}+$ & Sensitivity & Specificity & $\mathrm{LR}+$ & Sensitivity & Specificity & $\mathrm{LR}+$ \\
\hline Nsp/Hyp & 23 & 87 & 1.8 & 40 & 90 & 4.0 & 47 & 97 & 14 \\
\hline Nsp/MA & 57 & 87 & 4.3 & 77 & 90 & 7.7 & 100 & 97 & 30 \\
\hline Нур/МА & 57 & 77 & 2.4 & 77 & 60 & 1.9 & 100 & 53 & 2.1 \\
\hline
\end{tabular}

Values for sensitivity and specificity are given in \%; LR+, positive likelihood ratio; Nsp, normal spermatogenesis; Hyp, hypospermatogenesis; MA, maturation arrest. 
to the cut offs values for early spermatogonia and dividing/differentiating spermatogonia (Fig. 2). The individual evaluation of each patient revealed that patients with histologically $\mathrm{Nsp}(n=29)$ or patients with Hyp $(n=14)$ showed a high efficiency of meiotic entry and normal numbers of early spermatogonia or dividing/differentiating spermatogonia (Fig. 2A). Remarkably, only patients with histologically Nsp (seven from seven) as well as patients with Hyp (seven
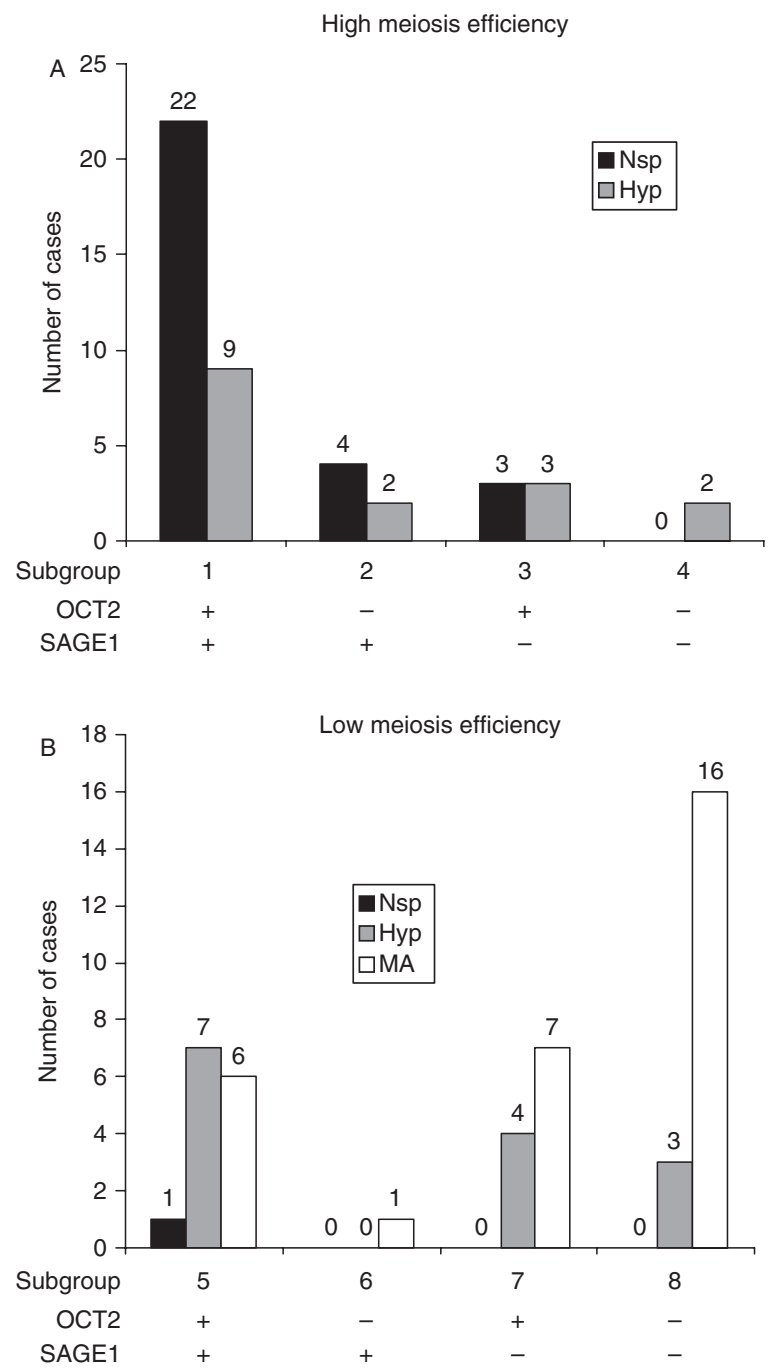

Figure 2 Classification of patients into high meiosis efficiency (A) and low meiosis efficiency (B). Both groups consist of four subgroups with normal numbers of early spermatogonia $\left(\mathrm{OCT}_{2}{ }^{+}\right.$) and dividing/ differentiating spermatogonia $\left(\mathrm{SAGE}^{+}\right.$) or reduced numbers of early spermatogonia $\left(\mathrm{OCT}_{2}{ }^{-}\right.$) and dividing/differentiating spermatogonia $\left(\mathrm{SAGE}^{-}{ }^{-}\right.$). The cut offs values from Table 3 were used for classification. Only cases with normal spermatogenesis ( $\mathrm{Nsp}$ ) or hypospermatogenesis (Hyp) demonstrated high meiosis efficiency (A). Interestingly, only Nsp or Hyp could compensate low numbers of early spermatogonia $\left(\mathrm{OCT}^{-}{ }^{-}\right.$) and/or dividing/differentiating spermatogonia (SAGE1 ${ }^{-}$) with a highly efficient meiosis (A). In contrast, all cases with maturation arrest (MA) showed low meiosis efficiency besides defects in numbers of early spermatogonia or dividing/differentiating spermatogonia (B). from 14) could compensate low numbers of spermatogonia with a high efficiency of meiotic entry (Fig. 2). In contrast, patients with MA always showed a low efficiency of meiotic entry and also revealed defects more often in the numbers of dividing/differentiating spermatogonia compared with defects in early spermatogonia (Fig. 2B). Especially subgroup 5 indicates missing compensatory mechanisms in meiosis as patients with normal numbers of spermatogonia showed low efficiency of meiosis (Fig. 2B).

\section{Discussion}

Besides the identification and evaluation of markers for early spermatogenesis, the primary aim of this study was the individual classification of defects in the early phase of spermatogenesis in cases of impaired spermatogenesis.

Intriguingly, the expression of OCT2 was found primarily in clusters of human $A_{\text {dark }}$ spermatogonia in a stem cell niche most often adjacent to the interstitial vasculature. This suggests that OCT2 is expressed very early in human spermatogenesis, most likely in stem cells as described by Lim et al. However, as also shown by Lim et al. (2011), as well as in this study, only a subgroup of $A_{\text {dark }}$ spermatogonia is OCT2-positive. In this study, identification of few OCT2-positive $A_{\text {pale }}$ spermatogonia is in accordance to results obtained in monkeys (Hermann et al. 2010), which showed that this subgroup also consists of undifferentiated spermatogonia, possibly stem cells. Thus, we believe like Dym et al. (2009) and (Hermann et al. 2010) that both $A_{\text {dark }}$ and $A_{\text {pale }}$ spermatogonia contain spermatogonial stem cells. In contrast, Ehmcke et al. (2006) described that only the $A_{\text {dark }}$ spermatogonia comprise spermatogonial stem cells.

In line with our previous findings (Hentrich et al. 2011), we found reduced numbers of spermatogonia in cases with MA. Moreover, we observed low numbers of early spermatogonia in cases with Hyp as well as in MA, indicating deficiencies already in the early phase of spermatogenesis. Thus, without $A_{\text {dark }}$ spermatogonia, which contribute to stem cell renewal, spermatogenesis is severely impaired (Holstein 1999).

In addition to stem cell markers, we were also interested in identification of marker(s) for dividing and differentiating spermatogonia. We observed SAGE1 expression in very few $A_{\text {dark }}$ spermatogonia, but mainly in dividing spermatogonia and (pre)-leptotene spermatocytes indicating entry into meiosis. Similarly Chen et al. (2011) and Lim et al. (2011) found SAGE1 in dividing spermatogonia and meiotic entry. Interestingly, SAGE1 expression was not detectable before puberty (Lim et al. 2011), thus further corroborating the importance of SAGE1 for differentiation of spermatogonia.

We found that SAGE1-positive spermatogonia were severely reduced in patients with Hyp and MA, but only 
rarely in patients with histologically Nsp. Thus, defects in the early phase of spermatogenesis, especially during mitosis, contribute also to impaired spermatogenesis. Similarly Bar-Shira Maymon et al. (2003) found a significant reduction in dividing spermatogonia in patients with spermatogenic defects, which was also described for Hyp and patients with MA (Steger et al. 1998). However, because reduced numbers of early spermatogonia might contribute to reduced numbers of mitotic spermatogonia, thus, any study on mitotic defects must also include quantification of early spermatogonia in order to discriminate properly between mitotic defects or reduced numbers of spermatogonia before meiosis or a combination of both.

SMAD3, which is also a key mediator of activininduced proliferation of Sertoli cells (Itman et al. 2011), is mainly expressed in pachytene spermatocytes in rat (Xu et al. 2003), mouse (Itman et al. 2011), and human testis (Hentrich et al. 2011). The reduced SMAD3positive pachytene spermatocyte numbers in patients with Hyp or MA (Hentrich et al. 2011) was also described without marker expression analysis in cases with reduced sperm cell numbers (Roosen-Runge et al. 1957, Zukerman et al. 1978). In contrast to the retained SMAD3 protein expression in cases with impaired spermatogenesis, the BOULE protein (expressed in human leptotene to pachytene spermatocytes in Nsp) was completely absent in cases with MA (Luetjens et al. 2004). Furthermore, alternative splicing of the BOULE gene was supposed to serve as a predictive marker for meiotic efficiency (Kostova et al. 2007).

The individual classification of patients with impaired spermatogenesis by categorizing meiotic efficiency in the early phase of spermatogenesis is a promising extension of the classification system reported by Bergmann \& Kliesch (1998, 2010). Although studies are lacking to assess the early phase of spermatogenesis up to pachytene, earlier studies showed up to $50 \%$ reduced recombination rates in cases with MA (Gonsalves et al. 2004, Egozcue et al. 2005). Similarily, a sixfold increased frequency of genetic abnormalities was observed in cases of MA compared with Hyp (Weedin et al. 2011).

In this study, we found that patients with histologically Nsp showed the lowest and patients with MA the highest numbers of defects in the early phase of spermatogenesis as well as in the meiotic phase. Especially in the subgroups 2-4 (Fig. 2A), a high meiotic efficiency but low numbers of spermatogonia, meiotic compensatory mechanisms must exist to ameliorate these defects. Obviously, all cases with MA are lacking this mechanism in addition to severely reduced numbers of spermatogonia. Interestingly, in cases with MA, reduction in early spermatogonia numbers $(54 \%)$ is nearly similar to the reduced numbers of dividing/differentiating spermatogonia $(42 \%)$, suggesting that loss of early spermatogonia might be primarily causative.
Of note, in cases of Hyp, which is the most heterogenous group (Takagi et al. 2001), we could identify both groups of patients with high or low efficiency of meiosis in almost all combinations with reduced numbers of early spermatogonia or reduced numbers of dividing/differentiating spermatogonia. However, in contrast to cases with MA, the frequency of low efficiency of meiosis was clearly lower in cases of Hyp.

In summary, this study revealed the efficiency of meiotic entry as a factor for male infertility and allows for a more detailed analysis of individual cases. We provide a refinement of the established classification system launched by Bergmann \& Kliesch (1998, 2010). In line with the diagnosis of MA, all cases demonstrated reduced numbers of spermatocytes; however, the majority additionally experienced reduced numbers of spermatogonia. Furthermore, we could provide evidence that in cases with Nsp as well as in cases with Hyp, compensatory meiosis mechanisms might exist to attenuate the loss of spermatogonia.

\section{Declaration of interest}

The authors declare that there is no conflict of interest that could be perceived as prejudicing the impartiality of the research reported.

\section{Funding}

This work was supported by a grant from the Deutsche Forschungsgemeinschaft to L Konrad (KFO 181/2 P2).

\section{Acknowledgements}

The authors thank Cornelia Hof for excellent technical assistance and Prof. Kliesch (Center of Andrology and Reproductive Medicine, University of Muenster, Germany) for biopsy sampling.

\section{References}

Bar-Shira Maymon B, Yogev L, Yavetz H, Lifschitz-Mercer B, Schreiber L, Kleiman SE, Botchan A, Hauser R \& Paz G 2003 Spermatogonial proliferation patterns in men with azoospermia of different etiologies. Fertility and Sterility 80 1175-1180. (doi:10.1016/S0015-0282(03)02161-7)

Bergmann M \& Kliesch S 1998 Hodenbiopsie. In Andrologie, pp 66-71. Eds W Krause \& W Weidner. Stuttgart, Germany: Enke-Verlag.

Bergmann M \& Kliesch S 2010 Testicular biopsy and histology. In Andrology, 3rd edn 3, pp 155-168. Eds E Nieschlag, HM Behre \& S Nieschlag. Berlin, Heidelberg, Germany: Springer-Verlag.

Brugh VM III \& Lipshultz LL 2004 Male factor infertility: evaluation and management. Medical Clinics of North America 88 367-385. (doi:10. 1016/S0025-7125(03)00150-0)

Cerilli LA, Kuang W \& Rogers D 2010 A practical approach to testicular biopsy interpretation for male infertility. Archives of Pathology \& Laboratory Medicine 134 1197-1204. (doi:10.1043/2009-0379-RA.1)

Chen YT, Chiu R, Lee P, Beneck D, Jin B \& Old LJ 2011 Chromosome $\mathrm{X}$-encoded cancer/testis antigens show distinctive expression patterns in developing gonads and in testicular seminoma. Human Reproduction $\mathbf{2 6}$ 3232-3243. (doi:10.1093/humrep/der330) 
Chiarini-Garcia H, Hornick JR, Griswold MD \& Russell LD 2001 Distribution of type A spermatogonia in the mouse is not random. Biology of Reproduction 65 1179-1185. (doi:10.1095/biolreprod65.4.1179)

Chiarini-Garcia H, Raymer AM \& Russell LD 2003 Non-random distribution of spermatogonia in rats: evidence of niches in the seminiferous tubules. Reproduction 126 669-680. (doi:10.1530/rep.0. 1260669)

Dadoune JP 2007 New insights into male gametogenesis: what about the spermatogonial stem cell niche? Folia Histochemica et Cytobiologica 45 $141-147$.

Dohle GR, Elzanaty S \& van Casteren NJ 2012 Testicular biopsy: clinical practice and interpretation. Asian Journal of Andrology 14 88-93. (doi:10.1038/aja.2011.57)

Dym M, Kokkinaki M \& He Z 2009 Spermatogonial stem cells: mouse and human comparisons. Birth Defects Research. Part C, Embryo Today: Reviews 87 27-34. (doi:10.1002/bdrc.20141)

Egozcue J, Sarrate Z, Codina-Pascual M, Egozcue S, Oliver-Bonet M, Blanco J, Navarro J, Benet J \& Vidal F 2005 Meiotic abnormalities in infertile males. Cytogenetic and Genome Research 111 337-342. (doi:10.1159/000086907)

Ehmcke J, Wistuba J \& Schlatt S 2006 Spermatogonial stem cells: questions, models and perspectives. Human Reproduction Update 12 275-282. (doi:10.1093/humupd/dmk001)

Gonsalves J, Sun F, Schlegel PN, Turek PJ, Hopps CV, Greene C, Martin RH \& Pera RA 2004 Defective recombination in infertile men. Human Molecular Genetics 13 2875-2883. (doi:10.1093/hmg/ddh302)

Hentrich A, Wolter M, Szardening-Kirchner C, Lüers GH, Bergmann M, Kliesch S \& Konrad L 2011 Reduced numbers of Sertoli, germ, and spermatogonial stem cells in impaired spermatogenesis. Modern Pathology 24 1380-1389. (doi:10.1038/modpathol.2011.97)

Hermann BP, Sukhwani M, Hansel MC \& Orwig KE 2010 Spermatogonial stem cells in higher primates: are there differences from those in rodents? Reproduction 139 479-493. (doi:10.1530/REP-09-0255)

Holstein AF 1999 Human spermatogenesis: basic research and clinical issues. Annals of Anatomy 181 427-436. (doi:10.1016/S0940-9602(99)80019-3)

Itman C, Wong C, Hunyadi B, Ernst M, Jans DA \& Loveland KL 2011 Smad3 dosage determines androgen responsiveness and sets the pace of postnatal testis development. Endocrinology 152 2076-2089. (doi:10. 1210/en.2010-1453)

Konrad L, Scheiber JA, Völck-Badouin E, Keilani MM, Laible L, Brandt H, Schmidt A, Aumüller G \& Hofmann R 2007 Alternative splicing of TGF- $\beta$ s and their high-affinity receptors T $\beta R I, T \beta R I I$ and T $\beta R I I I$ (Betaglycan) reveal new variants in human prostatic cells. BMC Genomics 8 318. (doi:10.1186/1471-2164-8-318)

Kostova E, Yeung $\mathrm{CH}$, Luetjens $\mathrm{CM}$, Brune $\mathrm{M}$, Nieschlag E \& Gromoll J 2007 Association of three isoforms of the meiotic BOULE gene with spermatogenic failure in infertile men. Molecular Human Reproduction 13 85-93. (doi:10.1093/molehr/gal101)

Lim J, Goriely A, Turner GD, Ewen KA, Jacobsen GK, Graem N, Wilkie AO \& Rajpert-De Meyts E 2011 OCT2, SSX and SAGE1 reveal the phenotypic heterogeneity of spermatocytic seminoma reflecting distinct subpopulations of spermatogonia. Journal of Pathology 224 473-483. (doi:10.1002/path.2919)

Looijenga LH 2011 Spermatocytic seminoma: toward further understanding of pathogenesis. Journal of Pathology 224 431-433. (doi:10.1002/ path.2939)

Luetjens CM, Xu EY, Rejo Pera RA, Kamischke A, Nieschlag E \& Gromoll J 2004 Association of meiotic arrest with lack of BOULE protein expression in infertile men. Journal of Clinical Endocrinology and Metabolism 89 1926-1933. (doi:10.1210/jc.2003-031178)

de Rooij DG 2009 The spermatogonial stem cell niche. Microscopy Research and Technique 72 580-585. (doi:10.1002/jemt.20699)

Roosen-Runge EC, Marberger E \& Nelson WO 1957 Quantitative investigations on human testicular biopsies. II. Infertility and other conditions. Fertility and Sterility 8 203-219.

Shetty G \& Meistrich ML 2007 The missing niche for spermatogonial stem cells: do blood vessels point the way? Cell Stem Cell 1 361-363. (doi:10. 1016/j.stem.2007.09.013)

Steger K, Aleithe I, Behre H \& Bergmann M 1998 The proliferation of spermatogonia in normal and pathological human seminiferous epithelium: an immunohistochemical study using monoclonal antibodies against Ki-67 protein and proliferating cell nuclear antigen. Molecular Human Reproduction 4 227-233. (doi:10.1093/molehr/4.3.227)

Takagi S, Itoh N, Kimura M, Sasao T \& Tsukamoto T 2001 Spermatogonial proliferation and apoptosis in hypospermatogenesis associated with nonobstructive azoospermia. Fertility and Sterility $\mathbf{7 6}$ 901-907. (doi:10.1016/S0015-0282(01)02732-7)

Weedin JW, Bennett RC, Fenig DM, Lamb DJ \& Lipshultz LI 2011 Early versus late maturation arrest: reproductive outcomes of testicular failure. Journal of Urology 186 621-626. (doi:10.1016/j.juro.2011. 03.156)

Xu J, Beyer AR, Walker WH \& McGee EA 2003 Developmental and stagespecific expression of Smad2 and Smad3 in rat testis. Journal of Andrology 24 192-200.

Yoshida S, Sukeno M \& Nabeshima Y 2007 A vasculature-associated niche for undifferentiated spermatogonia in the mouse testis. Science 317 1722-1726. (doi:10.1126/science.1144885)

Zukerman Z, Rodriguez-Rigau LJ, Weiss DB, Chowdhury AK, Smith KD \& Steinberger E 1978 Quantitative analysis of the seminiferous epithelium in human testicular biopsies, and the relation of spermatogenesis to sperm density. Fertility and Sterility 30 448-455.

Received 28 May 2014

First decision 13 June 2014

Revised manuscript received 18 June 2014

Accepted 26 June 2014 